\title{
Stochastic System Model Evaluated with First and Second Order Filters
}

\author{
Karen Alicia Aguilar Cruz, Romeo Urbieta Parrazales, José de Jesús Medel Juárez \\ Instituto Politécnico Nacional, Centro de Investigación en Computación, \\ Ciudad de México, Mexico
}

karen_ali320@hotmail.com,rurbieta700@gmail.com,jjmedelj@yahoo.com.mx

\begin{abstract}
This paper presents two stochastic filters considering autoregressive models of first and second order for parameter estimation and system identification. Each model is applied to a reference of the corresponding order and their recursive and non-recursive estimation results are compared; obtaining their error functional values to determine their performance. Due to the recursive methods give better approximation results, than the non-recursive ones, they are applied to describe the behaviour of the wind, which is a stochastic signal useful in the aerodynamic field, comparing the tracking results through off the functional error and the surroundings of the relative frequency histograms; including also a computational complexity graphic. To conclude, the second order filter has a better convergence performance at the expense of a higher computational cost, its pros and cons are mentioned. Nevertheless, choosing the filter order depends on its application.
\end{abstract}

Keywords: Estimation algorithms, recursive estimation, moment method, ARMA model, filtering techniques.

\section{Introduction}

Stochastic Systems (SS) have a great upswing in diverse scientific areas such as Biology, Physics and Chemistry [1]; where different techniques have been developed in order to give solutions to systems that can be modeled in an approximate way [2-4]. In past years, these systems have a greater relevance to deterministic models because they provide a much better characterization of the reality of the phenomena, including their uncertainty. In other areas like Administration, SS are used to determine the risk value of assets [5]; on the other hand, in Meteorology are applied to generate reliable models that allow the prediction of weather conditions with a bounded level of uncertainty [6-7].

Within the area of Control and Processing of Signals, SS look for the optimization of systems with random variables through their analysis [8-11], leading to the linear and non-linear Stochastic Filtering; being the linear Stochastic Filter with one or two delays, the model of interest for this paper. 
Basic linear systems are those that can be modeled like a Black Box (BB), where only the inputs and outputs of the system can be measured, while its internal characteristics cannot be obtained directly [12-13]. The model considered in this work corresponds to a deterministic system with Gaussian noise described through an AutoRegressive Moving Average (ARMA) model [14], leading to Gaussian linear stochastic systems, which are models that give response to BB systems through a parameter adjustment that allows the convergence to a reference system response.

Once the estimation is obtained it is necessary to determine the estimator algorithm performance by the comparison between the real and the identified responses by means of the functional error, which is a measure of how similar a signal is to a reference and how they converge to each other

Even when different filters have been designed, the question leads in which one is the best for a specific problem. For example, it will not be the same to identify the signals from a noisy turbulent wind to determine the angle variations of a flap in an airplane, than the temperature variations inside a home fridge. In some cases, the velocity of reaction is an important aspect, while in others the estimation or identification could be limited by hardware characteristics.

Within the present paper, two stochastic linear models of first and second order are presented. The Second Probability Moment (SPM) method was used [15] with finite difference equation to obtain their recursive forms for parameter estimation according to the stochastic ARMA selected model.

Then, for each different order model, their non-recursive and recursive descriptions are compared in an estimation task, determining the seconds lead to better results and these are applied to an identification example.

The example consists on determine the wind behavior, which is a practical case useful in Meteorology for obtaining weather conditions; in Aeronautic field due to the wind speed and direction are important parameters to map air routes; and in Control area, for obtaining the parameters an Unmanned Aerial Vehicle (UAV) needs for tracking trajectories tasks. Finally, the comparison of both models give as conclusion, criteria parameters for choosing one or the other in future applications.

\section{Recursive First Order Stochastic Model}

The representation of a BB system is given by (1) and (2), considering an ARMA (1, 1) stochastic model, with one input, one delay and evolution time $k \in Z_{+}$.

$$
\begin{gathered}
x_{k}=a_{1} x_{k-1}+b w_{k-1}, \\
y_{k}=c x_{k}+d v_{k},
\end{gathered}
$$

where, for an instant of time $k, x_{k} \in R$ is the state variable, $\left\{y_{k}\right\} \subseteq N\left(\mu_{y_{k}}, \sigma^{2} y_{k}<\infty\right)$ is the output variable, $\left\{w_{k}\right\} \subseteq N\left(\mu_{w_{k}}, \sigma^{2}{ }_{w_{k}}<\infty\right)$ is a stochastic system excitation, $\left\{v_{k}\right\} \subseteq N\left(\mu_{v_{k}}, \sigma_{v_{k}}^{2}<\infty\right)$ is a perturbation of bounded 
sequence, and $a_{1}, b, c, d \in R_{[-1,1]}$ are constants. According to the BB description, only $y_{k}$ and $w_{k}$ are measurable from the original system. So that, to initialize the model, not measurable variables and constants should be considered positive close to zero values, due to parameters are generally normalized acquiring values between 0 and 1 to avoid instability [16-18].

Beginning with (1) and (2) is obtained the recursive output $\hat{y}_{k}$ (3), having (4) as a generalized noise described by the noises that affect the system:

$$
\begin{gathered}
\tilde{y}_{k}=a_{1} \tilde{y}_{k-1}+\tilde{v}_{k}, \\
\tilde{v}_{k}=-d a_{1} v_{k}+b w_{k-1}+d v_{k} .
\end{gathered}
$$

Applying the second probability moment to (3), and using the expectation operator properties according to [19], it is obtained (5):

$$
\widetilde{a}_{1, k}=\left(E\left\{\widetilde{y}_{k} \widetilde{y}_{k-1}\right\}-d E\left\{\widetilde{v}_{k-1} \widetilde{y}_{k-1}\right\}\right) /\left(E\left\{\widetilde{y}_{k-1}^{2}\right\}\right)
$$

and the summarized description (6),

$$
\tilde{a}_{1, k}=P_{k} / Q_{k},
$$

where $P_{k}=\left(\widetilde{y}_{k} \tilde{y}_{k-1}\right)-d_{k}\left(\widetilde{v}_{k-1} \widetilde{y}_{k-1}\right)+(k-1) P_{k-1}$ and $Q_{k}=\widetilde{y}_{k}^{2}+(k-1) Q_{k-1}$ are the stationary functions which define the parameter (7):

$$
\widetilde{a}_{1, k}=\left[\widetilde{y}_{k} \widetilde{y}_{k-1}-d \widetilde{v}_{k-1} \widetilde{y}_{k-1}+(k-1) P_{k-1}\right] /\left[\widetilde{y}_{k-1}^{2}+(k-1) Q_{k-1}\right] .
$$

Taking into account in (7) that a) $\widetilde{a}_{1, k}$ is stationary while $P_{k-1}$ is described by $\widetilde{a}_{1, k-1} Q_{k-1}$ and, b) the denominator is described only by $Q_{k}$; the estimated stochastic parameter in its recursive form is (8).

$$
\widetilde{a}_{1, k}=\left[(k-1) Q_{k-1} / k Q_{k}\right] \widetilde{a}_{1, k-1}+\widetilde{y}_{k} \widetilde{y}_{k-1} / k Q_{k}-d \widetilde{v}_{k-1} \widetilde{y}_{k-1} / k Q_{k} .
$$

So that, the identified output (9) could be based on the estimation (8), which includes an implicit recursion and the use of an innovation process; allowing $\widetilde{v}_{k}$ to be approximated in distribution to a random variable $\widetilde{v}_{k}$, such that $\mu\left(\widetilde{v}_{k}\right) \approx \mu\left(\widetilde{v}_{k}\right)$, $\sigma\left(\widetilde{v}_{k}\right) \approx \sigma\left(\widetilde{v}_{k}\right)$ and the distribution function $\left\{\widetilde{v}_{k}\right\} \subseteq N\left(\mu_{\widetilde{v}_{k}}, \sigma_{\widetilde{v}_{k}}^{2}<\infty\right)$.

$$
\hat{y}_{k}=\widetilde{a}_{1, k} \widetilde{y}_{k-1}+\widetilde{v}_{k}
$$

The convergence error is based on the parameter estimation (8). The functional error is a recursive error measure which considers the second probability moment and allows obtaining the precision of an estimation algorithm in a probability sense. In a symbolic 
way, from $\widetilde{e}_{k}=a_{1}-\widetilde{a}_{1, k}$, the estimation functional error $\widetilde{J}_{k}=E\left\{\widetilde{e}_{k} \widetilde{e}_{k}\right\}$ is presented recursively in (10):

$$
\widetilde{J}_{k}=(1 / k)\left[\widetilde{e}_{k}^{2}+(k-1) \widetilde{J}_{k-1}\right]
$$

\section{Recursive Second Order Stochastic Model}

The stochastic second order model, with two delays and one input (ARMA $(2,1)$ ) is described by (11) and (12),

$$
\begin{gathered}
x_{k}=a_{1, k} x_{k-1}+a_{2, k} x_{k-2}+b w_{k-1}, \\
y_{k}=c x_{k}+d v_{k},
\end{gathered}
$$

where variables $x_{k}, y_{k}, w_{k}$ and $v_{k}$; as well as constants $a_{1}, b, c, d$ and now $a_{2}$; have the same characteristics and initializing considerations than those defining (1) and (2).

Expanding (11) and (12) is obtained the noise set (13) giving place to the recursive stochastic output (14), with two parameters to estimate ( $\widetilde{a}_{1, k}$ and $\widetilde{a}_{2, k}$ ).

$$
\begin{gathered}
\tilde{v}_{k}=-a_{1, k} d_{k} v_{k-1}-a_{2, k} d_{k} v_{k-2}+b c w_{k-1}+d v_{k}, \\
\tilde{y}_{k}=a_{1, k} \widetilde{y}_{k-1}+a_{2, k} \widetilde{y}_{k-2}+\widetilde{v}_{k} .
\end{gathered}
$$

Applying the second moment method to (14) and considering expressions in (15)

$$
\begin{gathered}
P_{k, 1}=(1 / k)\left[\left(\tilde{y}_{k} \tilde{y}_{k-1}-\tilde{v}_{k} \tilde{y}_{k-1}\right)+(k-1) P_{k-1,1}\right], \\
P_{k, 2}=(1 / k)\left[\left(\tilde{y}_{k} \tilde{y}_{k-2}-\tilde{v}_{k} \tilde{y}_{k-2}\right)+(k-1) P_{k-1,2}\right], \\
Q_{k, 1,1}=(1 / k)\left[\left(\tilde{y}_{k-1} \tilde{y}_{k-1}\right)+(k-1) Q_{k-1,1,1}\right], \\
Q_{k, 1,2}=(1 / k)\left[\left(\tilde{y}_{k-1} \tilde{y}_{k-2}\right)+(k-1) Q_{k-1,1,2}\right], \\
Q_{k, 2,1}=(1 / k)\left[\left(\tilde{y}_{k-1} \tilde{y}_{k-2}\right)+(k-1) Q_{k-1,1,2}\right], \\
Q_{k, 2,2}=(1 / k)\left[\left(\tilde{y}_{k-2} \tilde{y}_{k-2}\right)+(k-1) Q_{k-1,2,2}\right],
\end{gathered}
$$

we obtain the stochastic parameters (16) and (17), based on the second probability moment

$$
\begin{gathered}
\tilde{a}_{1, k}=P_{k, 1}\left(Q_{k, 1,1}\right)+P_{k, 2}\left(Q_{k, 2,1}\right)-d \tilde{v}_{k-1} \tilde{y}_{k-1} / k Q_{k, 1,1}, \\
\tilde{a}_{2, k}=P_{k, 1}\left(Q_{k, 1,2}\right)+P_{k, 2}\left(Q_{k, 2,2}\right)-d \tilde{v}_{k-1} \tilde{y}_{k-2} / k Q_{k, 2,2} .
\end{gathered}
$$

And their recursive forms (18) and (19):

$$
\tilde{a}_{1, k}=\left[(k-1) Q_{k-1,1,1} / k Q_{k, 1,1}\right] \tilde{a}_{1, k-1}+\tilde{y}_{k} \tilde{y}_{k-1} / k Q_{k, 1}-d \tilde{v}_{k-1} \tilde{y}_{k-1} / k Q_{k, 1,1},
$$




$$
\tilde{a}_{2, k}=\left[(k-1) Q_{k-1,2,2} / k Q_{k, 2,2}\right] \tilde{a}_{2, k-1}+\tilde{y}_{k} \tilde{y}_{k-2} / k Q_{k .2,2}-d \tilde{v}_{k-1} \tilde{y}_{k-2} / k Q_{k, 2,2} .
$$

The identified output (14) is based on the estimation made by (18) and (19), with an explicit recursion and the use of an innovation process that allows it to be approximated in distribution by a random variable $\widetilde{v}_{k}$, having $\mu\left(\widetilde{v}_{k}\right) \approx \mu\left(\widetilde{v}_{k}\right), \sigma\left(\widetilde{v}_{k}\right) \approx \sigma\left(\widetilde{v}_{k}\right)$ and $\left\{\widetilde{v}_{k}\right\} \subseteq N\left(\mu_{\widetilde{v}_{k}}, \sigma_{\widetilde{v}_{k}}^{2}<\infty\right)$ characteristics. Thus, the recursive stochastic output for the ARMA $(2,1)$ is determined by $(20)$ :

$$
\tilde{\tilde{y}}_{k}=\tilde{a}_{1, k} \tilde{\tilde{y}}_{k-1}+\tilde{a}_{2, k} \tilde{\tilde{y}}_{k-2}+\tilde{v}_{k} .
$$

On the other hand, comparing the two estimated parameters, considering the recursive errors (21) and (22),

$$
\begin{gathered}
\tilde{e}_{k, 1}=a_{1}-\tilde{a}_{1, k}, \\
\tilde{e}_{k, 2}=a_{2}-\tilde{a}_{2, k}
\end{gathered}
$$

leads to the functional errors (23) and (24)

$$
\begin{aligned}
& \widetilde{J}_{k, 1}=(1 / k)\left\lfloor\widetilde{e}_{k, 1}^{2}+(k-1)^{2} \widetilde{J}_{k-1,1}\right\rfloor, \\
& \widetilde{J}_{k, 2}=(1 / k)\left\lfloor\widetilde{e}_{k, 2}^{2}+(k-1)^{2} \widetilde{J}_{k-1,2}\right\rfloor .
\end{aligned}
$$

\section{Simulation of the First and Second Order Estimation Models}

The behaviors of the presented models are analyzed through the parameters they estimate and their corresponding functional error as a criterion to measure their performance. Each different order filter is probed in their recursive and non-recursive forms to determine which of both versions is better. Parameters are normalized and then dimensionless, on the other hand, as the evolution time of the software was not measured, graphics are presented in function of number of iterations; the time in seconds will depend on the software characteristics.

To probe the first order model, it is necessary to have a first order reference signal, which in this case is created from a known constant parameter of value between 0 and 1 , in this case closer to 1 . Fig. 1 shows the estimated parameters compared to the reference. It is possible to see a faster convergence when using the recursive description and in both cases, it is obtained a smoothed approximation, so that, the recursive description is better. This statement also could be proved by their errors functional, which are shown in Fig. 2, where even they are similar it is possible to identify the error obtained recursively converges faster to zero.

Similarly, but now considering two reference parameters for the second order filter description; we obtain the parameter estimation from Fig. 3. In this figure, two constant parameters valued between 0 and 1, close to the bounds, are tryed to be approximated 
simultaneously by using the second order recursive and non-recursive filter. The sintonization of more than one parameter is not an easy task because both parameters are related, in Fig. 3 only the second parameter is close to be approximated while the other reminds stationary to a different region but close to the reference. Fig. 4 matched with this and also helps to determine the recursive descriptions are the best.

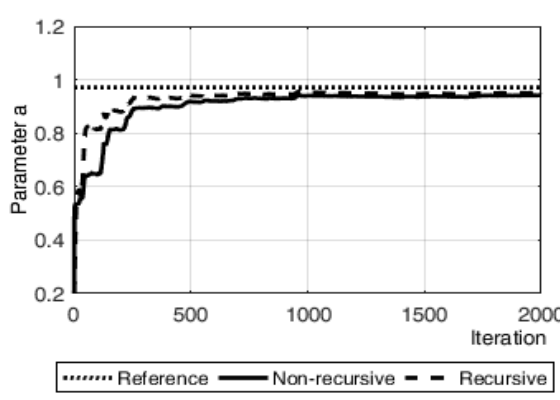

Fig. 1. Comparison of the normalized parameter $a$ estimated through the first order recursive and non-recursive model.
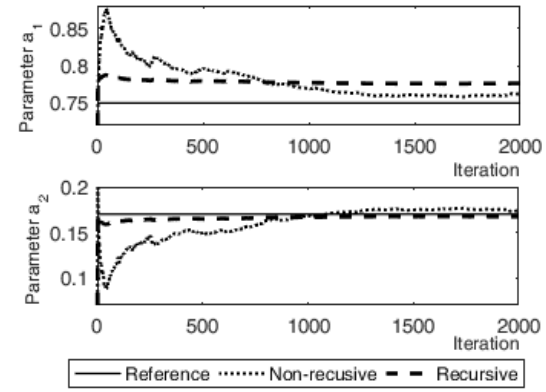

Fig. 3. Comparison of the normalized parameters $a_{1}$ and $a_{2}$ estimated through the second order recursive and non-recursive model.

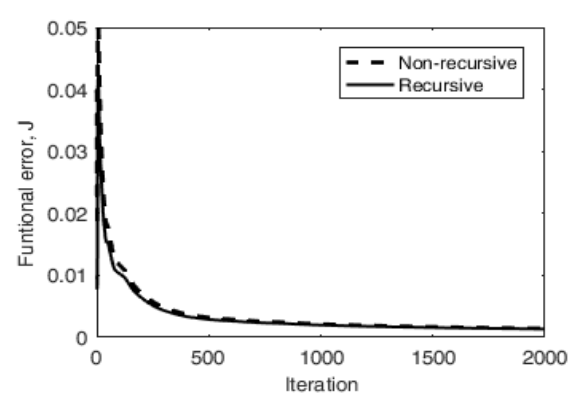

Fig. 2. Error functional from the parameters estimated through the recursive and nonrecursive first order model.

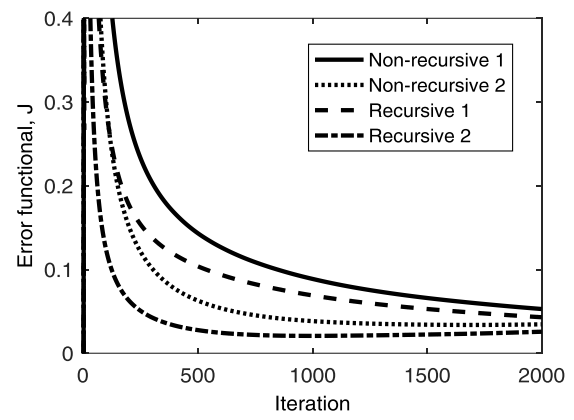

Fig. 4. Functional error of the parameters estimated through the recursive and nonrecursive second order model.

\section{$5 \quad$ Application Example and Results}

In the area of renewable energies, models that allow the estimation of wind variations are required in order to establish design limits for a better use, and the increase of security [20]. Within the wind energy, the models described in the present paper could be applied to identify the wind speed from a specific geographic region or the power variations given by this resource [21]. 
To identify the real wind speed [7] shown in Fig. 5 (left), which represents the variation of its velocity in $\mathrm{m} / \mathrm{s}$, thought a timeslot of $100 \mathrm{~s}$, both recursive stochastic models are used. The corresponding parameters are estimated and then applied in the identification models in order to compare their responses to the wind measured signal. The using of the recursive forms was taken because of the results obtained in Fig. 2 and Fig. 4, where the recursive parameter estimations, through the error functional, showed better performance than the non-recursive estimations.
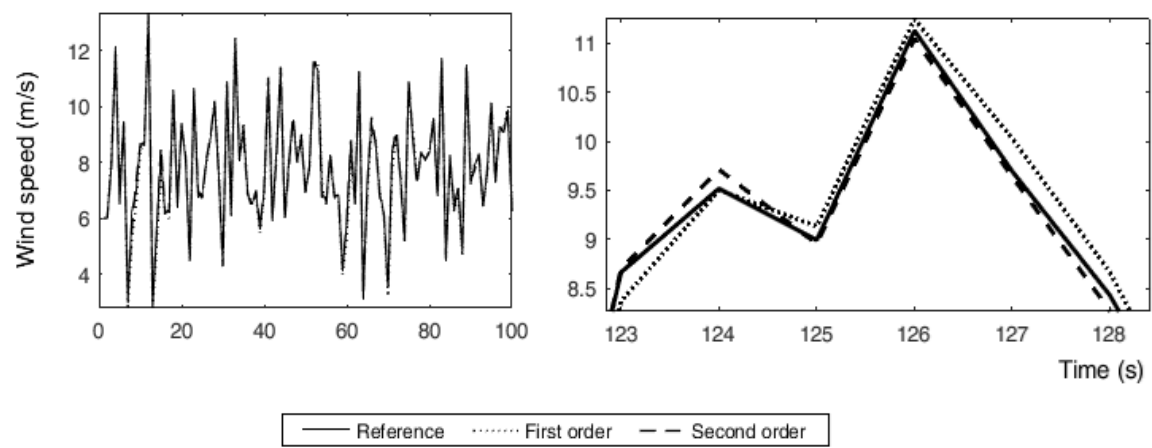

Fig. 5. Wind speed within an interval $t=100 \mathrm{~s}$, signal considered as a reference (left) and their estimation using first and second order methods (right).

The wind signal identifications, using both recursive models, are compared in Fig. 5 (right), where due to the large number of points defining the signal, it was considered only a smaller timeslot in order to visualize better the convergences. From Fig. 5 is possible to see the second order model has a faster convergence and better response to the shifting points in the signal; however, the first order model has visually a smoother performance, what is expected because of the model order. So that, for smooth variations a first order identification is acceptable.

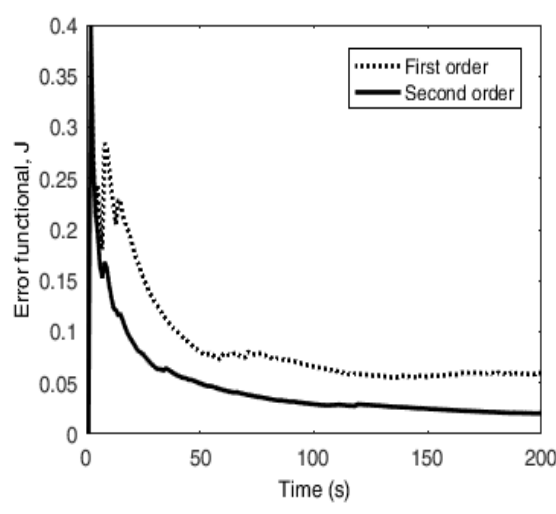

Fig. 6. Functional error of the output estimated signals, using the first and second order recursive methods.

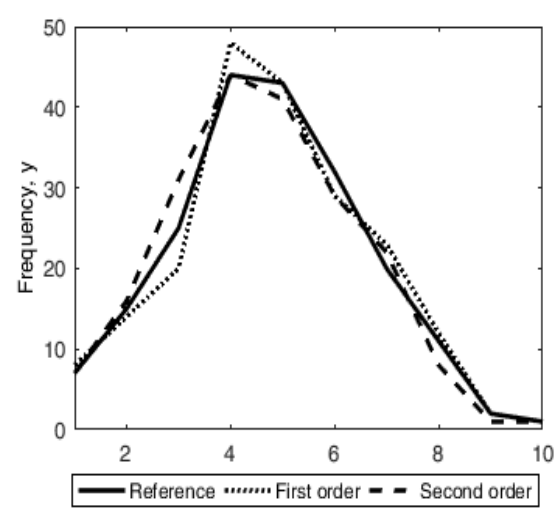

Fig. 7. Histogram of the output reference and the obtained estimations; using the recursive first and second order model. 
A comparison between the parameter estimation and the system parameters cannot be done because the second ones are unknown, what means, it is only possible to determine the performance of the model through their identified outputs and the functional error from their output signals. Fig. 6 is the representation of the functional error obtained from the error between the output reference and the identified output signal, giving a better idea of the convergence each method has. In this figure is best seen the performance differences that was not obvious in Fig. 5.

To know if the identified output ranges corresponds to the reference range, Fig. 7 was obtained, which is the relative frequency histogram of the wind speed. From the Fig. 7 is possible to see that even with a similar distribution, the second order model produces a better envelope of the histogram.

When performing an extensive simulation of 100 repetitions of identification of the wind reference signal, using both recursive methods; and considering different initial conditions giving the stochastic sense to the system; it is observed that the obtained functional error by the identified signals cover a convergence region, as shown in Fig. 8 , being this region more defined and with a faster convergence when using the second order model.
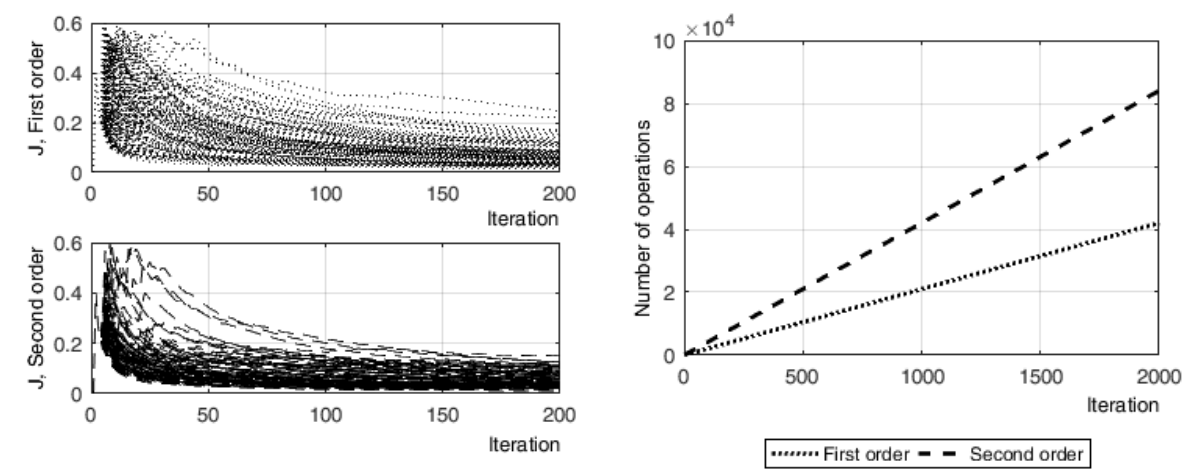

Fig. 8. Functional error obtained for the first and second order estimation methods; proof

Fig. 9. Algorithm complexity of identifiers of first and second order. made with 100 different stochastic signals.

Another characteristic which helps to compare both filters is the number of operations required to obtain an identification result. Fig. 9 displays how many the total number of operations needed are, when the iteration number increases through time.

\section{Conclusions}

The present paper describes two models with different order, first and second, to estimate stochastic parameters in a recursive and non-recursive form. According to the results, both methods have favorable characteristics, allowing the selection about which one of them is adequate for a specific system, being the recursive versions of both orders, the best. 
Respect to the estimated parameters, the second order model has better approximation when not smooth variation is presented, but, it is more difficult to initialize the estimation and it was seen that only one parameter converges adequately. Nevertheless, the first order one generates more acceptable results when the parameters do not vary considerable and the approximation is easier.

Both models accomplish the objective of approximate the reference signal from the estimation of the parameters that affect the recursive model of the identifiers. However, although the second order model could be considered more accurate, its complexity is also bigger while having to tune two parameters simultaneously, instead of only one. Hence, for digital implementations, it could be more convenient the first order model.

Finally, both models analyzed have characteristics that made them useful in systems modeled as a Black-Box (BB), with stationary bounded input-output conditions; and the analysis made in this paper allows the reader to have in mind the advantages of using one or other filter order, according to its necessities.

\section{References}

1. Britton, T., House, T., Lloyd, A. L., Mollison, D., Riley, S., Trapman, P.: Five challenges for stochastic epidemic models involving global transmission. Epidemics, 10, pp. 54-57 (2015)

2. Lecca, E. R., Puente, M. M.: Aplicaciones computacionales de las ecuaciones diferenciales estocásticas. Industrial Data, 9(1), pp. 064-075 (2006)

3. Mauricio, J. A.: Análisis de series temporales. Universidad Complutense de Madrid (2007)

4. Chernov, A., Debussche, A., Nobile, F.: Numerical methods for random and stochastic partial differential equations. Stochastics and Partial Differential Equations Analysis and Computations, 4(1), pp. 1-2 (2016)

5. Vitoriano, B.: Modelos y métodos de simulación estocástica. Aplicación en la valoración de opciones financieras.

6. Franzke, C. L., O'Kane, T. J., Berner, J., Williams, P. D., Lucarini, V.: Stochastic climate theory and modeling. Wiley Interdisciplinary Reviews: Climate Change, 6(1), pp. 63-78 (2015)

7. Emejeamara, F. C., Tomlin, A. S., Millward-Hopkins, J. T.: Urban wind: Characterisation of useful gust and energy capture. Renewable Energy, 81, pp. 162-172 (2015)

8. Ishizuka, S., Kajiwara, I.: Online adaptive PID control for MIMO systems using simultaneous perturbation stochastic approximation. Journal of Advanced Mechanical Design, Systems, and Manufacturing, 9(2), JAMDSM0015-JAMDSM0015 (2015)

9. Stojanovic, V., Nedic, N.: Robust Kalman filtering for nonlinear multivariable stochastic systems in the presence of non-Gaussian noise. International Journal of Robust and Nonlinear Control, 26(3), pp. 445-460 (2016)

10. Ramos, A., Cerisola, S.: Optimización estocástica. Pontificia Universidad COMILLAS de Madrid (2005)

11. Bartocci, E., Bortolussi, L., Nenzi, L., Sanguinetti, G. : System design of stochastic models using robustness of temporal properties. Theoretical Computer Science, 587, pp. 3-25 (2015)

12. Juárez, M., de Jesús, J., Zagaceta Álvarez, M. T., Palma Orozco, R.: Identificación con estimación para sistemas tipo caja negra. Revista Facultad de Ingeniería Universidad de Antioquia, (72), pp. 35-46 (2014) 
13. Kristensen, N. R., Madsen, H., Jørgensen, S. B.: Parameter estimation in stochastic greybox models. Automatica, 40(2), pp. 225-237 (2004)

14. Yaffee, R. A., McGee, M.: An introduction to time series analysis and forecasting: with applications of SAS® and SPSS®. Academic Press (2000)

15. Mátyás, L.: Generalized method of moment's estimation (Vol. 5). Cambridge University Press (1999)

16. Carayannis, G., Manolakis, D., Kalouptsidis, N.: A fast sequential algorithm for leastsquares filtering and prediction. IEEE transactions on acoustics, speech, and signal processing, 31(6), 1394-1402 (1983)

17. Hägglund, T., Åström, K. J.: Supervision of adaptive control algorithms. Automatica, 36(8), pp. 1171-1180 (2000)

18. Meng, L., Li, L., Veres, S. M.: Aerodynamic parameter estimation of an unmanned aerial vehicle based on extended Kalman filter and its higher order approach. In: Advanced Computer Control (ICACC), 2nd International Conference, Vol.5, pp. 526-531, IEEE (2010)

19. Medel, J., Urbieta, P., García, I.: Estimación de parámetros concentrados de un proceso estocástico de segundo orden. Revista mexicana de física, 60(1), pp. 80-87 (2014)

20. Gauterin, E., Kammerer, P., Kühn, M., Schulte, H.: Effective wind speed estimation: Comparison between Kalman Filter and Takagi-Sugeno observer techniques. ISA transactions, 62, pp. 60-72 (2016)

21. Verdejo, H., Escudero, W., Kliemann, W., Awerkin, A., Becker, C.,Vargas, L.: Impact of wind power generation on a large scale power system using stochastic linear stability. Applied Mathematical Modelling (2016) 